\title{
BMJ Open Evaluation of new or repurposed treatments for COVID-19: protocol for the phase Ib/IIa DEFINE trial platform
}

Erin Gaughan (1) , ${ }^{1,2}$ Tom Quinn, ${ }^{1,2}$ Annya Bruce, ${ }^{1}$ Jean Antonelli, ${ }^{1}$ Vikki Young, ${ }^{1}$ Joanne Mair, ${ }^{1}$ Ahsan Akram, ${ }^{1,2}$ Nik Hirani, ${ }^{1,2}$ Oliver Koch, ${ }^{3}$ Claire Mackintosh, ${ }^{3}$ John Norrie, ${ }^{4}$ James W Dear, ${ }^{2,5}$ Kevin Dhaliwall ${ }^{1,2}$

To cite: Gaughan E, Quinn T, Bruce A, et al. Evaluation of new or repurposed treatments for COVID-19: protocol for the phase Ib/lla DEFINE trial platform. BMJ Open 2021;11:e054442. doi:10.1136/ bmjopen-2021-054442

- Prepublication history and additional supplemental material for this paper are available online. To view these files, please visit the journal online (http://dx.doi.org/10.1136/ bmjopen-2021-054442).

$E G$ and $T Q$ contributed equally.

$E G$ and $T Q$ are joint first authors.

Received 25 June 2021 Accepted 04 October 2021

Check for updates

(c) Author(s) (or their employer(s)) 2021. Re-use permitted under CC BY-NC. No commercial re-use. See rights and permissions. Published by BMJ.

For numbered affiliations see end of article.

Correspondence to Professor Kevin Dhaliwal; Kev.Dhaliwal@ed.ac.uk

\section{ABSTRACT}

Introduction COVID-19 is a new viral-induced pneumonia caused by infection with a novel coronavirus, SARS-CoV-2. At present, there are few proven effective treatments. This early-phase experimental medicine protocol describes an overarching and adaptive trial designed to provide safety data in patients with COVID-19, pharmacokinetic (PK)/pharmacodynamic (PD) information and exploratory biological surrogates of efficacy, which may support further development and deployment of candidate therapies in larger scale trials of patients positive for COVID-19.

Methods and analysis Define is an ongoing exploratory multicentre-platform, open-label, randomised study. Patients positive for COVID-19 will be recruited from the following cohorts: (a) community cases; (b) hospitalised patients with evidence of COVID-19 pneumonitis; and (c) hospitalised patients requiring assisted ventilation. The cohort recruited from will be dependent on the experimental therapy, its route of administration and mechanism of action. Randomisation will be computer generated in a 1:1:n ratio. Twenty patients will be recruited per arm for the initial two arms. This is permitted to change as per the experimental therapy. The primary statistical analyses are concerned with the safety of candidate agents as add-on therapy to standard of care in patients with COVID-19. Secondary analysis will assess the following variables during treatment period: (1) the response of key exploratory biomarkers; (2) change in WHO ordinal scale and National Early Warning Score 2 (NEWS2) score; (3) oxygen requirements; (4) viral load; (5) duration of hospital stay; (6) PK/PD; and (7) changes in key coagulation pathways.

Ethics and dissemination The Define trial platform and its initial two treatment and standard of care arms have received a favourable ethical opinion from Scotland $A$ Research Ethics Committee (REC) (20/SS/0066), notice of acceptance from The Medicines and Healthcare Products Regulatory Agency (MHRA) (EudraCT 2020-002230-32) and approval from the relevant National Health Service (NHS) Research and Development (R\&D) departments (NHS Lothian and NHS Greater Glasgow and Clyde). Appropriate processes are in place in order to be able to consent adults with and without capacity while following the necessary COVID-19 safe procedures. Patients without capacity could be recruited via a legal representative. Witnessed electronic consent of participants or their legal representatives following consent discussions

\section{Strengths and limitations of this study}

- The trial is as flexible as possible to ensure a broad range of patients can be recruited and candidate therapies can be added or removed as evidence emerges.

- The team are collecting real-world data of medications at an early stage of their use in COVID-19 across the full spectrum of disease, allowing the administration of different treatment formulations (inhaled vs oral vs intravenous).

- The simultaneous collection of clinical outcomes as well as exploratory endpoints including clinical biomarkers, flow cytometry, pharmacokinetic/pharmacodynamic and thromboelastography allows further characterisation and elucidation of the temporal immunoinflammatory cascade in COVID-19 to inform on future therapy selection.

- This is a phase $\mathrm{lb} / \mathrm{lla}$ platform study and thus the primary endpoint is clinical safety, therefore our anticipated numbers will be too small to allow for definitive data on efficacy.

- Define is an experimental medicine platform, currently restricted to three clinical sites and so the generation of data will be slower than that of larger platforms with access to a greater number of patients.

was established. The results of each study arm will be submitted for publication in a peer-reviewed journal as soon as the treatment arm has finished recruitment, data input is complete and any outstanding patient safety follow-ups have been completed. Depending on the results of these or future arms, data will be shared with larger clinical trial networks, including the Randomised Evaluation of COVID-19 Therapy trial (RECOVERY), and to other partners for rapid roll-out in larger patient cohorts. Trial registration number ISRCTN14212905, NCT04473053.

\section{INTRODUCTION}

COVID-19 is a new viral-induced pneumonia caused by infection with a novel coronavirus, SARS-CoV-2. This highly contagious, zoonotic infection was first identified in Wuhan, 
China, in late $2019^{1}$ and its emergence has led to a global pandemic with a significant impact on health, society and the economy.

The majority of those infected with SARS-CoV-2 have asymptomatic or mild infection; however, $10 \%-20 \%$ of nonimmunised individuals will require admission to hospital with hypoxaemic respiratory failure requiring oxygen therapy and possibly ventilatory support. ${ }^{2}$ Risk factors for severity include increased age, obesity and medical history such as diabetes and hypertension. ${ }^{3}$ Genetic predisposition is now known to play a role ${ }^{4}$ and much of the lung damage is driven by a surge of inflammatory mediators. Mortality rate among hospital inpatients is felt to be as high as $20 \% .^{5}$ By September 2021, over 4.5 million individuals worldwide have died of COVID19. To date, there are few proven treatments with significant impact on mortality, and despite multiple successful vaccination developments it is expected that the virus will become endemic in the population, with significant levels of disease activity for years to come.

Define is a phase Ib/IIa experimental medicine trial. This protocol describes an overarching and adaptive trial designed to provide safety, pharmacokinetic (PK)/pharmacodynamic (PD) information and exploratory biological surrogates of efficacy which may support further development and deployment of candidate therapies in larger scale trials of patients positive for COVID-19 receiving normal standard of care.

Given the spectrum of clinical disease, communitybased infected patients or hospitalised patients can be included. Products requiring parenteral administration will only be investigated in hospitalised patients. Patients will be divided into cohorts as described below. Participants may be recruited from all three of the study cohorts, depending on the experimental therapy, its route of administration and mechanism of action.

Candidate therapies can be added to the protocol and previous candidates removed from further investigation as evidence emerges. The trial will be monitored by an independent data monitoring committee (DMC) to ensure patient safety.

Each candidate cohort will include a small cohort of patients randomised to candidate therapy or existing standard of care management dependent on disease stage at entry. Each treatment arm will recruit up to 20-30 patients to provide safety data, and PK-PD profile of potential therapeutic agents against COVID-19.

As COVID-19 follows a variable clinical path in individual patients, the protocol is designed to enable inclusion of patients across the disease stages. The trial is intended to provide mechanistic data from patients receiving standard of care therapy and from patients treated with the therapy candidates. The study will enable delivery of PK information and effects of standard of care and candidate agents on surrogate biomarkers of the disease process and the specific drug target.

\section{METHODS AND ANALYSIS}

This protocol follows the Standard Protocol Items: Recommendations for Interventional Trials reporting guidelines. ${ }^{6}$

\section{Trial design}

Define is an exploratory multicentre-platform, open-label, randomised study. This experimental medicine platform trial encompasses early-phase studies, and the identification of major safety signals is the primary objective. Each candidate therapy will include a small cohort of patients randomised to the candidate therapy or existing standard of care management dependent on disease stage at entry. Twenty patients will be recruited per arm for the initial two arms. This is permitted to change as per the experimental therapy. Randomisation will be computer generated and patients randomised in a 1:1:1:n ratio using a minimisation procedure based on sex, age, body mass index and a history of diabetes.

The proposed 'hybrid' platform (multiple interventions) and basket-type (multiple phenotypes) randomised trial is early phase to investigate biomarker response relevant to demonstrating COVID-19 clinical activity in repurposed drugs. As such, formal sample size calculations which would be mandatory for a confirmatory phase III randomised trial are neither feasible nor appropriate. An indicative sample size, considering the comparison of one active drug against control, each with $\mathrm{n}=20$ per group, and assuming $5 \%$ missing data, would give $80 \%$ power at a one-sided $10 \%$ level of significance, using a two-sample t-test, to detect an effect size of 0.7 in the difference of means in the biomarker between active and control.

Usually, the biomarker will be a continuous measure and the treatment effect estimated via a linear model which will adjust for baseline covariates highly correlated with the primary outcome, including possibly the baseline measurement of the primary outcome biomarker.

The study will not be powered for subgroup analyses. Safety data will be analysed on the as-treated data set (anyone who initiated on randomised treatment) and will be presented descriptively. Randomisation will involve computer-generated minimisation using a ratio of 1:1:1 across arms and there is no blinding. There are multiple interventions across several cohorts on the COVID-19 pathway (from community based, to breathless in hospital, to ventilation with different intensities), with potentially different primary outcomes. Hence, it is not practicable to produce bespoke minimisation algorithms for every possibility, so instead we will base the minimisation algorithm on what is currently known about risk factors associated with admission to intensive care unit or death. The minimisation will include a random element (set at 20\%) to increase unpredictability of allocation.

\section{Cohorts}

Patients with confirmed SARS-CoV-2 infection with relevant COVID-19 symptoms or signs will be recruited into this trial. As SARS-CoV-2 has a range of clinical 
Cohort 1A

Cohort 1B

Cohort 2A

Cohort 2B

Cohort 3
Community (primary care) patients with confirmed COVID-19

Community (primary care) patients with confirmed COVID-19 with new changes on CXR or CT scan compatible with COVID-19 and deemed 'high-risk' of hospitalisation or death*

Hospitalised confirmed COVID positive patients with new changes on CXR or new changes on CT compatible with COVID-19 but not requiring supplemental oxygen,

Hospitalised confirmed COVID positive patients with:

new changes on CXR or new changes on CT compatible with COVID-19 and requiring supplemental oxygen

Hospitalised patients with confirmed COVID-19 requiring assisted ventilation (including non-invasive and mechanical ventilation)

Figure 1 Cohorts of patients considered for participation in Define. *High risk is defined as over 50 years of age with comorbidities. CXR, chest x-ray.

manifestations, representatives of three target patient cohorts will be included in this trial, as seen in figure 1 . Participants may be recruited from one or more of these cohorts, depending on the experimental therapy under investigation. While the study will approach patients with suspected COVID-19 and conduct screening assessments, only patients who are confirmed SARS-CoV-2 positive will be randomised.

In terms of future studies, we anticipate additional standard of care arms may be required, given the evolving nature of the treatment modalities in COVID-19 in response to other clinical trials.

\section{Eligibility criteria}

Eligible participants were hospital inpatients over the age of 16 with confirmed SARS-CoV-2 infection within 14 days of a positive test. Exclusion criteria include pregnancy, lactation and the inability to reliably take or tolerate modes of treatment delivery, or if the patient was receiving anticoagulation, antiplatelet therapies or potassiumsparing diuretics which could not reasonably be withheld. Patients were also excluded if they had a current or recent history of severe, uncontrolled cardiac disease (New York Heart Association Functional Classification class IV), diabetes mellitus, renal impairment (estimated glomerular filtration rate (eGFR) $<30$ or ongoing dialysis) or hepatic impairment (alanine transaminase (ALT) $>5$ $\times$ upper limit of normal), anaemia with an $\mathrm{Hb}<80$, platelets (PLT) $<50$, hyponatraemia with an $\mathrm{Na}<120$ or K+ $>5$.0. Coenrolment with a Clinical Trial of an Investigational Medicinal Product will not be permitted.

See online supplemental file 1 for the Define patient information sheet and consent form as an example of the information and standard consent form given to the participants.

\section{Concomitant treatment}

Any drug required for the normal clinical care of these patients will be permitted, although as-treatment assets are added to the Define platform interactions with candidate therapies and will be reviewed on a case-by-case basis.

\section{Adverse events}

Participants in all arms will be instructed to contact the research team at any time after consent to study participation if any symptoms develop. For all participants, all adverse events (AE) that occur from the time of consent until 90 days after the final dose of investigational medication will be recorded in the case report form or $\mathrm{AE}$ $\log$. In the case of an $\mathrm{AE}$, the investigator should initiate the appropriate treatment according to their medical judgement. Clinical data and disease progression will be documented via linkage to control participants' medical records. All AEs that are not related to the patient's underlying condition or clinical interventions will be recorded following consent.

\section{Patient and public involvement}

There is an active patient representative in the Define Trial Steering Group. They have provided important feedback on logistical issues raised during the course of the first two treatment arms. Their contribution and feedback is ongoing regarding the design of the next arm of the trial, and how information is presented to the potential patients. Results will be disseminated to study participants on request.

\section{Trial status}

The recruitment for the first two treatment arms and a standard of care arm of Define was completed in January 2021. The first patient was recruited in September 2020. Nafamostat mesylate, a synthetic protease inhibitor, and GB0139, an inhaled galectin-3 inhibitor, were examined. Further arms are currently in preparatory stages.

\section{Objectives and endpoints}

\section{Primary}

The primary outcome is to evaluate the safety and tolerability of candidate agents as add-on therapy to Standard of Care in patients with COVID-19. Safety will be assessed using daily:

- Haematological and biochemical safety laboratory investigations. 
Full blood count, urea, creatinine, sodium, potassium, alanine transaminase, aspartate transaminase, alkaline phosphatase, coagulation screen (including activated partial thromboplastin time, prothrombin time, International normalised ratio, fibrinogen), glucose.

- Physical examination (if clinically relevant).

- Vital signs.

Blood pressure, heart rate, respiratory rate, oxygen saturations, oxygen requirement, temperature.

- ECG readings.

- AEs.

\section{Secondary}

- To explore the PK/PD or appropriate surrogate of bioavailability of the proposed trial treatments in patients with COVID-19.

- Assess the response of key exploratory biomarkers during treatment period. Evaluate the change from baseline values for key exploratory biomarkers of target engagement for each treatment.

- To evaluate the improvement or deterioration of patients in each treatment arm using the WHO ordinal scale and National Early Warning Score 2 (NEWS2) score.

- To evaluate the number of oxygen-free days (eg, duration (days) of oxygen use and oxygen-free days).

- To evaluate ventilator-free days and incidence and duration of any form of new ventilation use. This will be measured in duration (days) of ventilation and ventilation-free days. Incidence of any form of new ventilation use and duration (days) of new ventilation use.

- Change in the ratio of the oxygen saturation to fraction of inspired oxygen concentration $\left(\mathrm{SpO}_{2} / \mathrm{FiO}_{2}\right)$. $\mathrm{SpO}_{2} / \mathrm{FiO}_{2}$ will be measured daily from first dose to day 15, hospital discharge or death.

- To evaluate SARS-CoV-2 viral load. Qualitative and quantitative PCR determination of SARS-CoV-2 in saliva samples while hospitalised on days $1,3,5,8,11$ and 15 and oropharyngeal/nasal swab on the same days if tolerated.

- To evaluate time to discharge and duration to discharge following treatment.

- To evaluate the use of renal dialysis or haemofiltration for each treatment arm.

\section{Follow-up}

All discharged patients will undergo follow-up to assess for AEs at 30, 60 and 90 days.

\section{Data collection and analysis}

The biomarker variables will be mostly continuous measures and the treatment effect estimated via a linear model which will adjust for baseline covariates highly correlated with the primary outcome, including possibly the baseline measurement of the primary outcome biomarker.
The study will not be powered for subgroup analyses and these will be exploratory on a limited number of subgroups prespecified in the study protocol.

Due to the small sample sizes there will be no formal adjustment for missing data, and the primary analysis set-appropriate for early-phase proof of signal studiescould be a suitably defined per-protocol set (eg, those that were compliant with their randomised medications).

Safety data will be analysed on the as-treated data set (anyone who initiated on randomised treatment) and will be presented descriptively.

The independent DMC will review accumulating data, unblinded to the randomised groups. Their first and foremost responsibility will be the safety of the participants, and the committee may terminate the study at any time on the grounds of safety.

\section{Data management}

The principal investigator is responsible for the quality of the data recorded at each investigator site.

All investigators and study site staff involved with this study must comply with the requirements of the appropriate data protection legislation (including where applicable the General Data Protection Regulation with regard to the collection, storage, processing and disclosure of personal information). Access to personal information will be restricted to individuals from the research team treating the participants, representatives of the sponsor(s) and representatives of regulatory authorities.

Computers used to collate the data will have limited access measures via user names and passwords.

Published results will not contain any personal data that could allow identification of individual participants.

Appropriate representatives from the patient community were involved in the design of the study and the participant-facing documents were reviewed and commented on. This was carried out over email and via online meetings. A suitably experienced lay patient representative is on the Trial Steering Committee.

\section{ETHICS AND DISSEMINATION}

The Define trial platform and its initial two treatment and standard of care arms have received a favourable ethical opinion from Scotland A Research Ethics Committee (REC) $(20 / \mathrm{SS} / 0066)$, notice of acceptance from The Medicines and Healthcare Products Regulatory Agency (MHRA) (EudraCT 2020-002230-32) and approval from the relevant National Health Service (NHS) Research and Development (R\&D) departments (NHS Lothian and NHS Greater Glasgow and Clyde).

For the Define trial, a number of ethical and safety considerations have been addressed.

- The Define trial recruits adults with severe COVID-19 symptoms who are often incapacitated. The selection and enrolment of adults with incapacity will take place within the legal framework described in Adults with Incapacity (Scotland) Act 2000 and Medicines 
for Human Use (Clinical Trials) Regulations 2004. During the pandemic, relatives were not allowed to visit the hospital so the majority of consent for adults with incapacity will be undertaken over the phone. This is a well-practised method of obtaining consent and will be undertaken following all necessary guidance and with a set of instructions to ensure the personal legal representative has all the appropriate information and safeguarding in place. In rare cases, if no appropriate personal legal representative can be found, and it is believed to be in the best interests of the patient, consent from a professional legal representative may be sought. This is a person not connected with the trial who is either the doctor primarily responsible for the patient's medical treatment or is a person nominated by the relevant healthcare provider.

- If potential participants feel unable to or the physical signing of the consent form could increase potential transmission of COVID-19, a consent form completed by a member of the research team and witnessed by an independent member of the staff was acceptable. This has so far not been used.

- Trial treatments have been extensively tested preclinically and in patients prior to Define. All necessary safety information regarding the treatments is available to the research team and will be referred to in case of any serious safety event. All risks relating to the treatments will be carefully explained to both patients and their representatives.

- Inclusion and exclusion criteria will carefully considered to avoid exposing patients to undue risk.

- All appropriate approvals will be in place prior to the start of recruitment.

Ownership of the data arising from this study resides with the study team. Scientific publications and the sharing of clinical data generated as part of this trial are crucial to better understanding COVID-19 and developing new treatments. As such, the results of each study arm will be submitted for publication in a peer-reviewed journal as soon as the treatment arm has finished recruitment, data have been cleaned and any outstanding patient safety follow-ups completed. Depending on the results of these or future arms, data will be shared with larger clinical trial networks, including Randomised Evaluation of COVID-19 Therapy trial (RECOVERY), and to other partners for rapid roll-out in larger patient cohorts.
Author affiliations

${ }^{1}$ Centre for Inflammation Research, Queen's Medical Research Institute, The University of Edinburgh, Edinburgh, UK

${ }^{2}$ Royal Infirmary of Edinburgh, NHS Lothian, Edinburgh, UK

${ }^{3}$ Regional Infectious Diseases Unit, NHS Lothian, Edinburgh, UK

${ }^{4}$ Edinburgh Clinical Trials Unit, The University of Edinburgh, Edinburgh, UK

${ }^{5}$ Centre for Cardiovascular Science, Queen's Medical Research Institute, The University of Edinburgh, Edinburgh, UK

Acknowledgements We would like to thank Louise Sanford, our patient representative, for her invaluable contribution to the DEFINE trial and her ongoing efforts to safeguard the well-being of patients and monitor the overall conduct of this trial.

Contributors KD, JWD, JN, AA, NH, OK and CM designed the study and contributed to the study protocol. EG, TQ, AB, JA, VY and JM contributed to the study protocol and all study-related approvals. EG and TQ wrote the manuscript. All authors reviewed and approved the manuscript.

Funding This work was supported by LifeArc.

Competing interests None declared.

Patient consent for publication Not required.

Provenance and peer review Not commissioned; externally peer reviewed.

Supplemental material This content has been supplied by the author(s). It has not been vetted by BMJ Publishing Group Limited (BMJ) and may not have been peer-reviewed. Any opinions or recommendations discussed are solely those of the author(s) and are not endorsed by BMJ. BMJ disclaims all liability and responsibility arising from any reliance placed on the content. Where the content includes any translated material, BMJ does not warrant the accuracy and reliability of the translations (including but not limited to local regulations, clinical guidelines, terminology, drug names and drug dosages), and is not responsible for any error and/or omissions arising from translation and adaptation or otherwise.

Open access This is an open access article distributed in accordance with the Creative Commons Attribution Non Commercial (CC BY-NC 4.0) license, which permits others to distribute, remix, adapt, build upon this work non-commercially, and license their derivative works on different terms, provided the original work is properly cited, appropriate credit is given, any changes made indicated, and the use is non-commercial. See: http://creativecommons.org/licenses/by-nc/4.0/.

ORCID iD

Erin Gaughan http://orcid.org/0000-0002-9295-5503

\section{REFERENCES}

1 Hu B, Guo H, Zhou P, et al. Characteristics of SARS-CoV-2 and COVID-19. Nat Rev Microbiol 2021;19:141-54.

2 Wiersinga WJ, Rhodes A, Cheng AC, et al. Pathophysiology, transmission, diagnosis, and treatment of coronavirus disease 2019 (COVID-19): a review. JAMA 2020;324:782-93.

3 Jordan RE, Adab P, Cheng KK. Covid-19: risk factors for severe disease and death. BMJ 2020;368:m1198-2.

4 Pairo-Castineira E, Clohisey S, Klaric L, et al. Genetic mechanisms of critical illness in COVID-19. Nature 2021;591:92-8.

5 Izcovich A, Ragusa MA, Tortosa F, et al. Prognostic factors for severity and mortality in patients infected with COVID-19: a systematic review. PLoS One 2020;15:e0241955.

6 Chan A-W, Tetzlaff JM, Gøtzsche PC, et al. SPIRIT 2013 explanation and elaboration: guidance for protocols of clinical trials. $B M J$ 2013;346:e7586. 